LAWRENCE LIVERMORE N A TION AL LABORATORY

\title{
UCRL-JRNL-200828
}

\section{Time-Reversal Analysis for Scatterer Characterization}

D. H. Chambers, J. G. Berryman

November 7, 2003

Physical Review Letters 
This document was prepared as an account of work sponsored by an agency of the United States Government. Neither the United States Government nor the University of California nor any of their employees, makes any warranty, express or implied, or assumes any legal liability or responsibility for the accuracy, completeness, or usefulness of any information, apparatus, product, or process disclosed, or represents that its use would not infringe privately owned rights. Reference herein to any specific commercial product, process, or service by trade name, trademark, manufacturer, or otherwise, does not necessarily constitute or imply its endorsement, recommendation, or favoring by the United States Government or the University of California. The views and opinions of authors expressed herein do not necessarily state or reflect those of the United States Government or the University of California, and shall not be used for advertising or product endorsement purposes. 


\title{
Time-Reversal Analysis for Scatterer Characterization
}

\author{
David H. Chambers* \\ University of California, Lawrence Livermore National Laboratory, \\ P.O.Box 808 L-154, Livermore, CA 94551-9900, USA \\ James G. Berryman ${ }^{\dagger}$ \\ University of California, Lawrence Livermore National Laboratory, \\ P.O.Box 808 L-200, Livermore, CA 94551-9900, USA
}

(Dated: November 8, 2003)

Only the simplest monopole scattering behavior has usually been treated in previous timereversal analyses. A new application of time-reversal processing of wave scattering data permits characterization of scatterers by analyzing the number and nature of the singular functions (or eigenfunctions) associated with individual scatterers when they have multiple contributions from monopole, dipole and/or quadrupole scattering terms. We discuss acoustic, elastic, and electromagnetic scattering problems for low frequencies $(k a<1, k$ being the wavenumber and $a$ the radius of the scatterer). Specific examples for electromagnetic scattering from one of a number of small conducting spheres show that each sphere can have up to six distinct timereversal eigenfunctions associated with it.

\section{LLNL Preprint: UCRL-JP-200828 November 7, 2003.}

PACS numbers: 41.20.Jb, 43.20.Fn, 81.05.Rm, 43.60.Pt

Previous work shows that time-reversal signal processing $[1,2]$ and analysis [3-5] of wave scattering data can be successfully used to achieve super-resolution in refocusing through random media [6] (i.e., better focusing than can be achieved in a homogeneous medium with the same size array) of waves onto the location of a source even when the actual location of that source is unknown. This technique has obvious applications to communications in complex environments such as ocean acoustics and urban cell phone reception and transmission. Timereversal processing has also been shown to produce good imaging of scatterers in random media [7,8] as long as the magnitude of the fluctuations in the random medium is not too large.

The great majority of time-reversal analysis done so far has concentrated on the simplest (i.e., monopole) scattering behavior. However, a different application of timereversal processing (and the one we will concentrate on here) involves characterizing scatterers by analyzing further the number and nature of the multiple singular functions (or eigenfunctions) associated with each scatterer including dipole and quadrupole, as well as the standard monopole, terms when all are present and detectable. For example, Chambers and Gautesen [9] and Chambers [10] have analyzed time-reversed acoustic scattering for fluids when there is contrast both in bulk modulus (monopole) and fluid density (dipole). They showed for the case of

* chambers2@llnl.gov

† berryman1@llnl.gov; Address for correspondence. a small fluid sphere that the orthogonality condition for the eigenfunctions in the aperture of the array constrains the dipole moment of the scatterer to three possible orthogonal orientations. Each eigenfunction is generated by a linear combination of scattering from the monopole moment and one of the three orientations of the dipole moment. The monopole plus the three orthogonal orientations of the dipole thus represent four independent degrees of freedom for the time-reversal array scattering from a fluid sphere. The maximum number of possible eigenvalues of the system is equal to the number of degrees of freedom (four). Fewer eigenvalues may be observed for special array and sphere configurations that possess additional symmetries (e.g., only three eigenvalues for a linear array). For larger fluid spheres, additional eigenvalues are generated as higher order scattering moments (e.g., quadrupole) become important [10]. The dependence of the eigenvalues and eigenfunctions on the scattering geometry, as well as mechanical properties of the sphere, could be used to characterize the sphere.

The observation that the number of eigenstates of a time-reversal system is bounded by the number of degrees of freedom (multipole moments and orientations) in the scattering can also be generalized to other wave systems. For example, an additional mode of scattering in elastic media is due to contrast in shear modulus (quadrupole, with from three to six relevant orientations, depending on the symmetry of the scattering problem). For isotropic media, there would be a single scalar associated with contrast in shear modulus, while for anisotropic media there could be as many as five. If $k$ is the wave number and $a$ is the radius of a spherical scatterer and 
we consider only small values of $k a$, this sort of analysis shows quite simply then that in acoustics and elasticity we expect a single eigenfunction for monopole scattering, as many as three distinct eigenfunctions for dipolar scattering, and from three to six more for quadrupole scattering for elastic wave time-reversal experiments in solids, and correspondingly (significantly) fewer modes in fluids.

In contrast, electromagnetic scattering does not permit monopole contributions, but dipole and higher order multipole contributions are possible. Dipolar contributions (as we show here) can come either from contrast in dielectric properties, or from conductivity. For poorly conducting materials, only the dielectric scattering modes are important. But, for highly conducting materials, both the dielectric and the conducting properties of the scatterer can contribute terms to the scattering matrix. Since the dipole is a vector quantity, there can be as many as three contributions from each type of scattering feature - six total. Our analysis takes a form similar to that of Tortel et al. [11], and we present a summary of it here.

Consider an array of $N$ short, crossed-dipole elements lying in the plane $z=-z_{a}$, where $z_{a}$ is the distance between the plane and the scattering sphere (located at the origin in $3 \mathrm{D}$ ). The position of the $n t h$ element is given by the vector $\mathbf{r}_{n}=\left(\xi_{n}, \eta_{n},-z_{a}\right)$. Following Krauss [12], the electric field at the field point $\mathbf{r}$ radiated from the $n t h$ element is given by

$\mathbf{E}_{n}^{(i)}\left(\mathbf{R}_{n}\right)=\frac{i k e^{i k R_{n}}}{4 \pi \varepsilon_{0} c R_{n}} \hat{\mathbf{R}}_{\mathbf{n}} \times\left[\hat{\mathbf{R}}_{\mathbf{n}} \times\left(d_{H} I_{n}^{H} \hat{\mathbf{e}}_{\mathbf{x}}+d_{V} I_{n}^{V} \hat{\mathbf{e}}_{\mathbf{y}}\right)\right]$,

where $c$ is the speed of light, $k$ is the wavenumber, $\epsilon_{0}$ is the electrical permittivity, and $\mathbf{R}_{n}=\mathbf{r}-\mathbf{r}_{n}$. The scalar $R_{n}$ is the magnitude of the vector $\mathbf{R}_{n}$ and $\hat{\mathbf{R}}_{\mathbf{n}}$ is the unit vector in the direction of $\mathbf{R}_{n}$. The horizontal and vertical dipoles in the element (lengths $d_{H}$ and $d_{V}$ ) are driven by the currents $I_{n}^{H}$ and $I_{n}^{V}$ respectively. The horizontal dipole is oriented parallel to the $x$ axis (unit vector $\hat{\mathbf{e}}_{\mathbf{x}}$ ) and the vertical dipole is oriented parallel to the $y$ axis (unit vector $\hat{\mathbf{e}}_{\mathbf{y}}$ ).

A sphere of radius $a \ll z_{a}$ is placed in front of the array, centered at the origin. The field incident on the sphere from the nth element can be approximated as a plane wave coming from the direction of the element. For a sphere much smaller than a wavelength $(k a \ll 1)$, the field scattered from an incident plane wave is given to leading order $\mathcal{O}\left(k^{3} a^{3}\right)[13]$ by

$$
\mathbf{E}^{(s)}(\mathbf{r})=-\frac{k^{2} e^{i k r}}{r}[\hat{\mathbf{r}} \times(\mathbf{m}+\hat{\mathbf{r}} \times \mathbf{p})],
$$

where $\mathbf{p}$ is the induced electric dipole moment and $\mathbf{m}$ is the induced magnetic dipole moment generated by the incident field. The moments are related to the incident field $E_{n}^{(i)}$ evaluated at the position of the sphere $\mathbf{r}=0$ $\left(\mathbf{R}_{n}=-\mathbf{r}_{n}\right)$ :

$$
\mathbf{m}=-m_{0} \hat{\mathbf{r}}_{n} \times \mathbf{E}_{n}^{(i)}\left(-\mathbf{r}_{n}\right), \quad \mathbf{p}=p_{0} \mathbf{E}_{n}^{(i)}\left(-\mathbf{r}_{n}\right),
$$

where $p_{0}=a^{3}\left(\tilde{n}^{2}-1\right) /\left(\tilde{n}^{2}+2\right), \tilde{n}^{2}=\varepsilon+i 4 \pi \sigma / \omega$, and $m_{0}=-i B_{1}^{m} / k^{3}$ (see [13]). The various factors are $\varepsilon$ the relative permitivity of the sphere, $\sigma$ the conductivity, $\omega$ the angular frequency, and $B_{1}^{m}$ is a quantity defined in reference [13] that determines the strength of the magnetic moment. When the conductivity of the sphere is small, $B_{1}^{m}$ can be neglected to leading order, so then the magnetic moment $\mathbf{m}$ does not contribute to the scattered field. In general, $p_{0}$ and $m_{0}$ are complex and can be represented in terms of magnitude and phase: $p_{0}=\left|p_{0}\right| e^{i \theta_{p}}$, $m_{0}=\left|m_{0}\right| e^{i \theta_{m}}$.

The scattered field induces voltages on each dipole of the array elements. From reference [14], the voltages induced on the dipoles of the $m t h$ element can be expressed as

$$
\begin{aligned}
& V_{m}^{H}=-d_{H}\left[\hat{\mathbf{r}}_{m} \times\left(\hat{\mathbf{r}}_{m} \times \hat{\mathbf{e}}_{\mathbf{x}}\right)\right] \cdot \mathbf{E}^{(s)}\left(\mathbf{r}_{m}\right), \\
& V_{m}^{V}=-d_{V}\left[\hat{\mathbf{r}}_{m} \times\left(\hat{\mathbf{r}}_{m} \times \hat{\mathbf{e}}_{\mathbf{y}}\right)\right] \cdot \mathbf{E}^{(s)}\left(\mathbf{r}_{m}\right) .
\end{aligned}
$$

Combining these with the previous expressions for the incident field (1) and scattered field (2), we can calculate the coupling between the voltages in the $m t h$ receiving element and the currents in the $n t h$ transmitting element generated from the scattering by the sphere. We define an operator (a $3 \times 3$ matrix) $\Delta_{m n}=\hat{\mathbf{r}}_{m} \cdot \hat{\mathbf{r}}_{n} \mathfrak{I}-\hat{\mathbf{r}}_{n} \hat{\mathbf{r}}_{m}^{T}$ (where $\mathfrak{I}$ is the identity matrix) to replace the double vector cross-products that appear repeatedly in these formulas, and note that this operator has a character similar to that of a projection operator. This observation greatly simplifies the resulting algebra. The details are elementary but still somewhat tedious, so we will not show them here. The heart of the scattering operator of interest has the general form $\mathbf{S} \equiv \Delta_{m m}\left(m_{0} \Delta_{m n}-p_{0} \Delta_{m m}\right) \Delta_{n n}$, and so it follows immediately that

$$
\mathbf{S}=m_{0} \Delta_{m n}-p_{0} \Delta_{m m} \Delta_{n n} .
$$

Then the $2 \times 2$ matrix that connects any source to any receiver is

$$
\left[\begin{array}{cc}
K_{m n}^{H H} & K_{m n}^{H V} \\
K_{m n}^{V H} & K_{m n}^{V V}
\end{array}\right] \equiv \frac{i k^{3} e^{i k\left(r_{m}+r_{n}\right)}}{4 \pi \varepsilon_{0} c r_{m} r_{n}}\left[\begin{array}{c}
d_{H} \hat{e}_{x}^{T} \\
d_{V} \hat{e}_{y}^{T}
\end{array}\right] \mathbf{S}\left[\begin{array}{ll}
d_{H} \hat{e}_{x} & d_{V} \hat{e}_{y}
\end{array}\right]
$$

and the final result is:

$$
\left[\begin{array}{c}
V_{m}^{H} \\
V_{m}^{V}
\end{array}\right]=\left[\begin{array}{cc}
K_{m n}^{H H} & K_{m n}^{H V} \\
K_{m n}^{V H} & K_{m n}^{V V}
\end{array}\right]\left[\begin{array}{c}
I_{n}^{H} \\
I_{n}^{V}
\end{array}\right]
$$

where the superscripts $H$ and $V$ refer to the horizontal and vertical dipoles in each element and the corresponding polarizations. (For more details see [15].) The $2 \times 2$ matrix $\mathbf{K}_{\mathbf{m n}}$ can then be written as

$$
\mathbf{K}_{\mathbf{m n}}=\frac{i k^{3} q}{4 \pi \varepsilon_{0} c} e^{i k\left(r_{m}+r_{n}\right)} \hat{\mathbf{K}}_{\mathbf{m n}},
$$

with the elements of $\hat{\mathbf{K}}_{\mathbf{m n}}$ determined by Eq. (6), where $q=\sqrt{\left|p_{0}\right|^{2}+\left|m_{0}\right|^{2}}$. Note that $\hat{\mathbf{K}}_{\mathbf{n m}}=\hat{\mathbf{K}}_{\mathbf{m n}}^{T}$ by reciprocity (superscript $T$ indicates the transpose). The diagonal elements of the $\hat{\mathbf{K}}_{\text {mn }}$ matrix describe the coupling between dipoles with the same polarizations. The 
off-diagonal elements describe the cross-polarization coupling. Thus, all combinations of polarization coupling are represented in $\hat{\mathbf{K}}_{\mathbf{m n}}$.

From these results, the relationship between the transmitted currents and the received voltages over the entire array can easily be constructed. Let $\mathbf{V}$ be the vector of received voltages and $\mathbf{I}$ the vector of transmitted currents (both with length $2 N$ ). Then we can write

$$
\mathbf{V}=\mathbf{T} \mathbf{I}
$$

where

$$
\begin{aligned}
\mathbf{V} & =\left[V_{1}^{H}, V_{1}^{V}, \ldots, V_{m}^{H}, V_{m}^{V}, \ldots, V_{N}^{H}, V_{N}^{V}\right]^{T}, \\
\mathbf{I} & =\left[I_{1}^{H}, I_{1}^{V}, \ldots, I_{n}^{H}, I_{n}^{V}, \ldots, I_{N}^{H}, I_{N}^{V}\right]^{T}
\end{aligned}
$$

and the $2 N \times 2 N$ matrix $\mathbf{T}$ is assembled from all the matrices $\mathbf{K}_{\mathbf{m n}}$. (The current vector $\mathbf{I}$ is not to be confused with the identity matrix $\mathfrak{I}$.) The matrix $\mathbf{T}$ is the inter-element response matrix (transfer matrix in acoustics [16]) and is symmetric (from reciprocity). It has units of impedance and can be considered the part of the radiation impedance of the array attributable to the presence of the sphere. Its $2 N \times 2 N$ size results from the two components of polarization for each element in the array. If only one polarization is used $\left(d_{H}=0\right.$ or $\left.d_{V}=0\right)$, three-quarters of the matrix elements are zero and $\mathbf{T}$ reduces to an $N \times N$ matrix.

Decomposition of the time-reversal operator (TRO) is achieved by first considering its definition $\mathbf{T}^{*} \mathbf{T}$. Its eigenvalues and eigenvectors then characterize the properties of the array as a time-reversal system. Each eigenvector represents a set of complex currents that, when applied to the dipoles in each element of the array, will produce a field that focuses on the sphere. In addition, the conjugates of the resulting voltages from the received field will be proportional to the transmitted currents. The constant of proportionality is the eigenvalue (square of the singular value) and measures the apparent strength of the scattering mode that is excited in the sphere by the incident field. Direct calculation of the eigenvalues and eigenvectors of $\mathbf{T}^{*} \mathbf{T}$ can be unwieldy, so the singular value decomposition (SVD) for $\mathbf{T}$ has often been used instead [5]. Since $\mathbf{T}$ is symmetric the SVD is [10] $\mathbf{T} \boldsymbol{\Phi}=\Lambda \boldsymbol{\Phi}^{*}$, where the singular values $\Lambda$ are real and non-negative. The singular vectors $\boldsymbol{\Phi}$ are also the eigenvectors of the TRO, and the squares of the singular values are the eigenvalues of the TRO. The SVD can be further simplified by factoring out common quantities. Then, letting $z_{j}=e^{-i k r_{j}}$, for $j=1, \ldots, N$, we define

$$
\boldsymbol{\Phi}=\frac{1}{\sqrt{i}}\left[\phi_{1} z_{1}, \phi_{2} z_{1}, \ldots, \phi_{2 N-1} z_{N}, \phi_{2 N} z_{N}\right]^{T}
$$

and $\Lambda=\frac{k^{3} q}{4 \pi \varepsilon_{0} c} \lambda$, then the SVD becomes

$$
\hat{\mathbf{T}} \phi=\lambda \phi^{*},
$$

where

$$
\hat{\mathbf{T}}=\left[\begin{array}{cccc}
\hat{\mathbf{K}}_{11} & \hat{\mathbf{K}}_{12} & \cdots & \hat{\mathbf{K}}_{1 \mathrm{~N}} \\
\hat{\mathbf{K}}_{21} & \hat{\mathbf{K}}_{22} & \cdots & \hat{\mathbf{K}}_{2 \mathrm{~N}} \\
\vdots & \vdots & \ddots & \vdots \\
\hat{\mathbf{K}}_{\mathrm{N} 1} & \hat{\mathbf{K}}_{\mathrm{N} 2} & \cdots & \hat{\mathbf{K}}_{\mathrm{NN}}
\end{array}\right]
$$

By factoring out the complex exponential from the original singular vectors $\Phi$, the part of the phase responsible for focusing the transmitted field on the sphere is eliminated. As shown by Chambers and Gautesen [9], this is common to all eigenvectors of the TRO in the presence of a single scatterer. The remaining vector $\phi$ represents a slowly varying (possibly complex) amplitude distribution over the array, which may have a pattern of nulls depending on the nature of the scattering from the sphere.

A careful deconstruction of the elements of the matrix $\hat{\mathbf{T}}=\hat{\mathbf{T}}_{p}+\hat{\mathbf{T}}_{m}$ reveals that it can be expressed as a sum of two terms (for dielectric and conducting contributions), each of which is an outer product of three vectors:

$$
\hat{\mathbf{T}}_{p}=-e^{i \theta_{p}}\left(\mathbf{g}_{1} \mathbf{g}_{1}^{T}+\mathbf{g}_{2} \mathbf{g}_{2}^{T}+\mathbf{g}_{3} \mathbf{g}_{3}^{T}\right)
$$

and

$$
\hat{\mathbf{T}}_{m}=e^{i \theta_{m}}\left(\mathbf{g}_{4} \mathbf{g}_{4}^{T}+\mathbf{g}_{5} \mathbf{g}_{5}^{T}+\mathbf{g}_{6} \mathbf{g}_{6}^{T}\right)
$$

where the vectors $\mathbf{g}_{j}$, for $j=1, \ldots, 6$, are known explicitly from the foregoing analysis. The singular vectors for matrices of this form can be expressed as linear combinations of the vectors $\mathbf{g}_{1}$ through $\mathbf{g}_{6}, \phi=\sum_{j=1}^{6} \gamma_{j} \mathbf{g}_{j}$. This fact reduces the SVD for the $2 N \times 2 N$ matrix $\hat{\mathbf{T}}$ (eq. 12) to an SVD of the $6 \times 6$ matrix $\mathbf{G}$ (a big reduction if $N$ is much larger than 3) having elements $G_{j l}=\mathbf{g}_{j}^{T} \mathbf{g}_{l}$ :

$$
\begin{aligned}
-e^{i \theta_{p}} \sum_{l=1}^{6} G_{j l} \gamma_{l} & =\lambda \gamma_{j}^{*}: j=1,2,3, \text { and } \\
e^{i \theta_{m}} \sum_{l=1}^{6} G_{j l} \gamma_{l} & =\lambda \gamma_{j}^{*}: j=4,5,6 .
\end{aligned}
$$

This representation of the inter-element transfer matrix as a sum of a small number of products of vectors also occurs for the acoustic case (see Refs. $[9,10]$ ). It is a consequence of the small number of terms used in the partial wave expansion for the scattered field (2). Here the scattered field is generated by an electric dipole moment and a magnetic dipole moment, each of which can be oriented in three mutually orthogonal directions. The two types of moments, each with three orthogonal directions, represent six degrees of freedom for the construction of the scattered field. This means that, for small $k a$, there are at most six eigenvectors of the time reversal operator, with each eigenvector generated by a linear combination of the fields produced by the induced electric and magnetic dipole moments.

The analytical solution of Eq. (16) for the singular vectors and singular values may be obtained in principle, but 


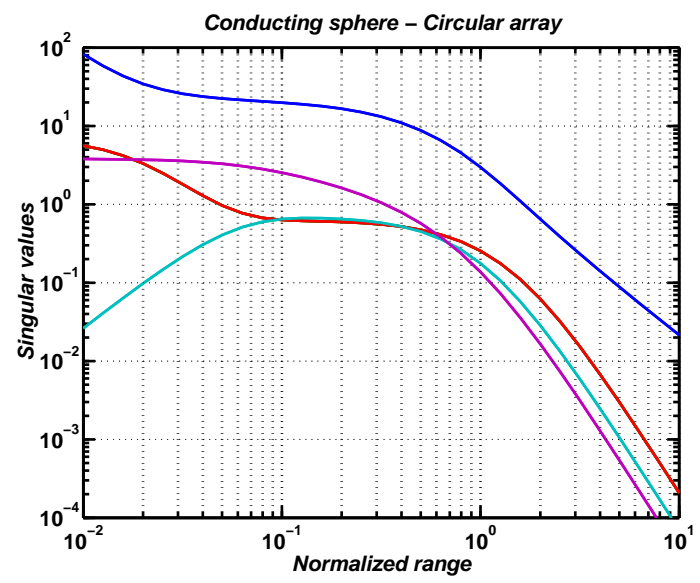

FIG. 1: Singular values for a conducting spherical scatterer as a function of normalized (by the array radius $D / 2$ ) range $z_{a}$. Cross-range for this figure is $x=0$. Although there are six singular functions for the conducting sphere, at $x=0$ two pairs of singular values are degenerate, so only four curves are visible here.

it would be exceedingly unwieldy and generally uninformative. Instead, we consider the solution when the array is symmetric about both the $\xi$ and $\eta$ axes. Then all the off-diagonal elements of the $\mathbf{G}$ matrix are zero except for $G_{14}$ and $G_{25}$ (since $\mathbf{G}$ is symmetric), which greatly simplifies the solution. Results for a circular array satisfying these conditions and having $d_{H}=d_{V}$ and array diameter $D$ are plotted in Figs. 1 and 2. Fig. 1 shows curves of singular values for a conducting spherical scatterer as a function of normalized range $2 z_{a} / D$. The value of cross-range for this case is $x=0$, i.e., the center of the array. Although there are six singular functions for the conducting sphere, at $x=0$ two pairs of singular values are degenerate, so only four distinct curves are visible. Fig. 2 shows the singular values for the same conducting spherical scatterer as a function of normalized crossrange $2 x / D$. Normalized range for this case is unity. Note that, although there are six singular functions for the conducting sphere, the top two singular values (for dielectric dipole modes) are degenerate because of the mutual symmetry between the sphere and the circular array.

For practical applications of these results, it is clear that, for a fixed range scenario and either stationary source/receiver array with moving target or stationary target and moving array, data such as those in Fig. 2 would also be readily available. Furthermore, using the top two (degenerate) singular values as the standard, and assuming at least $1 \%$ accuracy in singular value measurement/computation, we see that all the singular values would be detectable in principle at the center of the circular array, but two of them would decay very rapidly as the measurement position expanded from once to twice (or more) the radius of the array. This observation would provide some information about the cross-range location

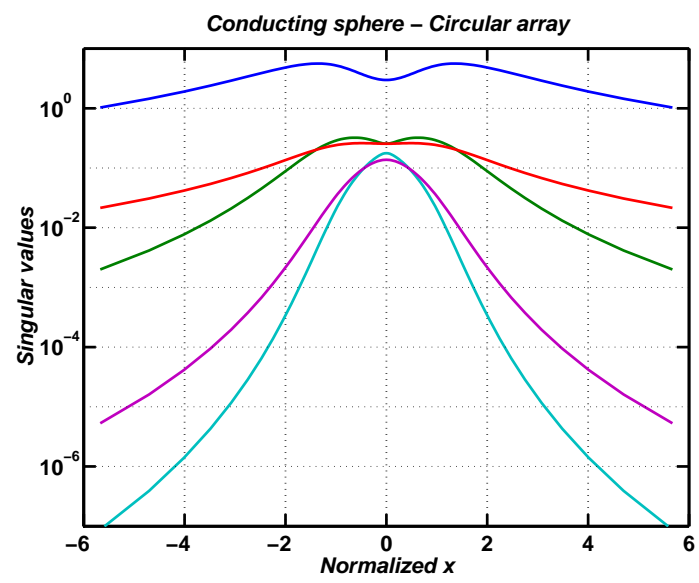

FIG. 2: Singular values for a conducting spherical scatterer as a function of normalized (again by the array radius $D / 2$ ) cross-range $x$. Normalized range for this figure is unity. Note that, although there are six singular functions for the conducting sphere, the top two singular values (for dielectric dipole modes) are degenerate because of the mutual symmetry of the sphere and the circular array.

of the scattering target.

In contrast, acquiring data like those in Fig. 1 would require either a moving target and/or non-stationary (or multiple) arrays in order to obtain information based on the different values of range from array to target. But again using the top singular value as the standard, we see that (at least at the array center) all three of the other distinct singular values stay within about two orders of magnitude (1\%) of the largest out to 10 times the array radius. This means that two widely separated arrays might be needed to measure the range to such a conducting target by analysis of the computed singular values, assuming that the arrays also have sufficient sensitivity and measurement accuracy. Of course, with two arrays so widely separated, it might be more efficient simply to use triangulation to detect the range, while the bearing of the target would always be straightforward to determine using traditional signal processing methods at each array. For very distant scatterers, only the highest two singular values (the two that are degenerate) would be detectable. This is reasonable as, for very distant targets, it becomes difficult to determine anything about the scatterer except its bearing from a single small array. But introduction of additional widely spaced arrays permits triangulation for target range, and by further combining the data from several arrays they could then be used to do scatterer characterization along the lines outlined here.

We find that relationships between symmetries of scattering and time-reversal eigenvalues seen previously in acoustics $[16,17]$ carry over to electromagnetics. Similar relationships will also hold for other wave scattering problems as well, such as poroelasticity [18]. 


\section{ACKNOWLEDGMENTS}

Work performed by University of California, Lawrence Livermore Laboratory, under the auspices of the U.S. Department of Energy under contract No. W-7405-ENG-48.

\section{REFERENCES}

[1] M. Fink, Physics Today 50, 34 (March, 1997).

[2] W. A. Kuperman and D. R. Jackson, in Imaging of Complex Media with Acoustic and Seismic Waves, edited by M. Fink, W. A. Kuperman, J.-P. Montagner, and A. Tourin (Springer-Verlag, Berlin, Germany, 2002), vol. 84 of Topics in Physics, pp. 43-96.

[3] D. R. Jackson and D. R. Dowling, J. Acoust. Soc. Am. 89, 171 (1991).

[4] D. R. Dowling and D. R. Jackson, J. Acoust. Soc. Am. 91, 3257 (1992).

[5] C. Prada, S. Manneville, D. Spoliansky, and M. Fink, J. Acoust. Soc. Am. 99, 2067 (1996).

[6] P. Blomgren, G. Papanicolaou, and H. Zhao, J. Acoust. Soc. Am. 111, 238 (2002).

[7] L. Borcea, G. C. Papanicolaou, C. Tsogka, and J. G. Berryman, Inverse Problems 18, 1247 (2002).
[8] J. G. Berryman, L. Borcea, G. C. Papanicolaou, and C. Tsogka, J. Acoust. Soc. Am. 112, 1509 (2002).

[9] D. H. Chambers and A. K. Gautesen, J. Acoust. Soc. Am. 109, 2616 (2001).

[10] D. H. Chambers, J. Acoust. Soc. Am. 112, 411 (2002).

[11] H. Tortel, G. Micolau, and M. Saillard, J. Electromagn. Waves Appl. 13, 687 (1999).

[12] J. D. Krauss, Antennas (McGraw-Hill, San Francisco, 1988), 2nd ed.

[13] M. Born and E. Wolf, Principles of Optics (Pergamon Press, New York, 1980), 6th ed.

[14] W. L. Stutzman and G. A. Thiele, Antenna Theory and Design (John Wiley and Sons, New York, 1981).

[15] D. H. Chambers and J. G. Berryman, Analysis of the time-reversal operator for a small sphere in an electromagnetic field (2003), LLNL preprint UCRLJC-152633, April 10, 2003.

[16] C. Prada and M. Fink, Wave Motion 20, 151 (1994).

[17] C. Bardos and M. Fink, Asymptotic Analysis 29, 157 (2002).

[18] J. G. Berryman, J. Math. Phys. 26, 1408 (1985). 